\title{
Kapitel 7.
}

\section{Einleitung zu Teil II}

Teil I dieses Leitfadens behandelt unter anderem eine Vielzahl verschiedener Formen der vollständigen Induktion. Ihnen allen ist gemeinsam, dass sämtliche Objekte, über die Aussagen gemacht werden, bezüglich einer gegebenen Nachbarschaftsbeziehung nur endlich weit von irgendwelchen Startobjekten entfernt sind. Objekte, die nicht in endlich vielen Schritten von Startobjekten aus erreichbar sind, können von diesen Beweismustern grundsätzlich nicht erfasst werden.

Der vorliegende Teil II des Leitfadens hat zum Hauptthema, diese Einschränkung zu überwinden, also eine Form der vollständigen Induktion zu entwickeln, die auch anwendbar ist, wenn Objekte unendlich weit von Startobjekten entfernt sind.

Dazu müssen zwei Teilaspekte berücksichtigt werden:

1. Man braucht eine Sammlung von Objekten mit einer Nachbarschaftsbeziehung, in der es überhaupt Objekte gibt, die unendlich weit von Startobjekten entfernt sind. In der Menge der natürlichen Zahlen mit Startobjekt 0 und der Nachfolgerbeziehung, zum Beispiel, gibt es solche Objekte ja gerade nicht: Jede natürliche Zahl ist in endlich vielen Nachfolgerschritten von der Null aus erreichbar.

2. Man braucht ein Beweismuster der vollständigen Induktion, das auch die Objekte mit unendlichem Abstand von Startobjekten erfasst. Dafür liegt es nahe, eines der bisherigen Beweismuster der vollständigen Induktion - das die endlich weit entfernten Objekte erfassen kann - um Fälle für die Objekte mit unendlichem Abstand zu erweitern.

Kapitel 8 soll zu diesem Hauptthema hinführen. Es rekapituliert einige Fakten über Folgen, Reihen und Grenzwerte und Zusammenhänge mit der vollständigen Induktion.

Das eigentliche Hauptthema teilt sich auf die nächsten beiden Kapitel 9 und 10 auf.

Kapitel 9 stellt die sogenannten Ordinalzahlen vor, zu denen geeignete Objekte für den obigen Teilaspekt 1 gehören.

Kapitel 10 hat das Beweismuster der transfiniten Induktion und damit den obigen Teilaspekt 2 zum Inhalt.

Zur Abrundung folgt in Kapitel 11 noch ein Exkurs über mathematisches Arbeiten, der unmittelbar an ein umfangreiches Beispiel in Kapitel 10 anknüpft. 